\title{
Synthesis, Characterizations and Antibacterial Studies of Conducting Poly Aminothiophenols
}

\author{
U. Anto Maria Jeraldin ${ }^{1}$ and R. Jaya Shanthi ${ }^{2 *}$ \\ PG \& Research Department of Chemistry, Auxilium College, Vellore, Tamil Nadu, India ${ }^{1,2}$
}

\begin{abstract}
The oxidative chemical polymerization of poly ortho, meta and Para Aminothiophenols were carried out in an aqueous hydrochloric acid using ammonium persulphate as an oxidant. The synthesized polymers were characterized using FT-IR, UV-VIS, XRD and SEM. The basic framework of benzenoid and quinoid stretching in synthesized polymers were confirmed by FTIR. The UV-VIS spectra of isomers of poly aminothiophenol showed two distinct absorption peaks for $\pi-\pi^{*}$ and $n-\pi^{*}$ transitions due to the benzenoid and quinoid rings. The thermal properties were carried out using TGA, DTA and DSC studies and found that the synthesized polymers were thermally stable even up to $750^{\circ} \mathrm{C}$. The SEM analysis clearly showed the morphology of the polymers and the morphology varied from polymer to polymer. The DC conductivity was carried out by electrochemical method and it showed that the polymers were semiconducting in nature with the order of $10^{-4}$. The antibacterial study carried out by well cut diffusion method indicated that the antibacterial activities varied depending on the type of bacterial strains and were compared with the standard Ciprofloxacin. The antibacterial activities were found to increase with increase in concentrations of polymers.
\end{abstract}

Keywords: Conducting Polymers, Chemical Oxidation, Aminothiophenol, Thermal Study, Conductivity, Antibacterial Study

\section{INTRODUCTION}

Conducting polymers have received considerable attention because of their interesting electronic, physical properties, chemical stability and their potential technological applications [1-4]. Conducting polymers are poly conjugated compounds, which are insulators in their pure state; but when treated with oxidizing or reducing agents can be converted into polymer salts having reasonable electrical conductivity. They have wide applications in devices such as solar cells, rechargeable batteries, light emitting diodes, micro-actuators, electro chromic displays, field effect transistors, sensors etc [5,6].

Polyaniline (PANI) is one of the most promising conducting polymers because of its unique properties like ease of preparation in aqueous medium, good stability in air, simplicity in doping, improved electronic properties, controllable by oxidation and protonation state, excellent environmental stability, moderately high conductivity in the doped state and Potential applications in electronic devices [7,8]. Polymers are typically utilized in electrical, optical and electronic devices as insulators because of their very high electrical resistivity. The dielectric properties of heterogeneous polymers [9] play an important role in device applications such as high performance capacitors, electrical cable insulation, electronic packaging etc [10,11].

A derivative of polyaniline, Amino Thio Phenols (ATP) are interesting electrochemical materials since thiol and amine have different re-activities [12-14], the effective use of this molecular assembly may give rise to unique morphologies leading to multi-purpose chemical strategies. Moreover, the aromatic (conjugated $\pi$-electron system) ring of ATP intensifies the electrical coupling. Therefore, they can show electrochemical behavior resembling anilines and phenols. In recent years, electrical, optical, and dielectric properties of conducting polymers like polyaniline and substituted polyaniline synthesized by chemical oxidation polymerization have been studied in great detail.

Conducting polymers having good biocompatibility combined with antibacterial and cytotoxicity property can lead to tremendous development in the field of biomedicine and controlled drug release, the latter of which can be tremendously useful in cancer treatment. A range of biomedical applications for conducting polymers were considered which includes development of artificial muscles, controlled drug release and stimulation of nerve regeneration [15].

In the present work, isomers of Poly Amino Thio Phenol (PATP) were synthesized by chemical oxidative polymerization method and were characterized by different spectroscopic techniques such as FT-IR, UV-VIS, XRD and SEM. The thermal stabilities of the synthesized polymers were analyzed using TGA/DTA and DSC studies. An attempt has been made to investigate the AC conductivity and antibacterial studies against gram positive bacteria like Staphylococcus aureus and gram negative bacteria like Escherichia coli were carried out for the chemically synthesised poly (ortho-, meta-, and para) aminothiophenol compounds. 


\title{
International Advanced Research Journal in Science, Engineering and Technology
}

\author{
Vol. 5, Issue 8, August 2018
}

\section{EXPERIMENTAL METHODS}

A. Materials: Monomer o-aminothiophenol (Alfa aezar), Ammonium persulfate (Sigma-Aldrich) and Hydrochloric acid were purchased from Merck Ltd., India. All other chemicals and reagents were of Analytical Grade and used as received without any further purification in the present study.

B. Synthesis of Poly (ortho Aminothiophenol): The poly (o-aminothiophenol) was synthesized by chemical oxidative polymerisation method in acidic medium. Ortho Aminothiophenol (monomer), Ammonium per sulphate (oxidant) were taken in the ratio of 1:2. The monomer was dissolved in $1 \mathrm{M}$ hydrochloric acid. The aqueous solution of the oxidising agent was slowly added into monomer solution. The solution was kept stirring for about $5 \mathrm{~h}$ at room temperature, after which the greenish powder obtained as residue. The resultant polymer was washed with distilled water until the filtrate was colorless then with acetone and with methanol to remove excess initiator, monomer and oligomers. Finally, the resultant precipitate was dried at room temperature for 24 hours [16]. Same procedure was adopted for the synthesis of isomers of poly (meta-, and para) aminothiophenol.

C. Characterization Techniques: The FT-IR spectra of the synthesized isomers of poly aminothiophenol (PATP) were performed using Perkin Elmer 1750 FTIR Spectrophotometer at room temperature. UV-VIS spectra were recorded from 200-800 nm using SHIMADZU model UV-2450 spectrophotometer by dissolving the polymers in DMSO as a solvent. TGA/DTA was recorded using Perkin Elmer Diamond under a nitrogen atmosphere up to $700^{\circ} \mathrm{C}$ at the heating rate of $20^{\circ} \mathrm{C} / \mathrm{min}$. The Differential Scanning Calorimetry (DSC) was measured using Mettler Toledo DSC 822e from room temperature to $500^{\circ} \mathrm{C}$. X-ray diffraction (XRD) was recorded with Bruker AXS D8 Advance diffractometer at room temperature at $2 \theta$ angle ranging from $0^{\circ}$ to $80^{\circ}$. The morphological study of the chemically synthesized poly (ortho-, meta-, and para) aminothiophenol compounds were carried out using Scanning Electron Microscopy, model: Jeol $6390 \mathrm{LV}$ with accelerating voltage of $0.5 \mathrm{kV}$ to $30 \mathrm{kV}$.

D. Electrical Conductivity Studies: Photochemical measurements were carried out on a CHI608E electrochemical workstation and a $100 \mathrm{~W}$ Xe arc lamp (OSRAM, Germany) was used as the light source. In the present study, standard three electrode cell, with a $\mathrm{Pt}$-wire was used as counter electrode, $\mathrm{Ag} / \mathrm{AgCl}$ in saturated $\mathrm{KCl}$ as a reference electrode and the synthesized polymers poly (aminothiophenol) were used as a working electrode and an aqueous solution of 0.1 $\mathrm{M} \mathrm{Na}_{2} \mathrm{SO}_{4}$ was used as an electrolyte. The working electrode was prepared by having about $5 \mathrm{mg}$ of sample was ground with $10 \mu \mathrm{L}$ of Triton $\mathrm{X}-100$ and $20 \mu \mathrm{L}$ of deionised water to make slurry. The slurry was spread on a $0.5 \times 0.5$ $\mathrm{cm}^{2}$ Fluorine-doped Tin Oxide (FTO) glass substrate with an active area of $0.25 \mathrm{~cm}^{2}$ by doctor blade method using scotch tape as spacer and then dried in hot plate for overnight.

E. Antibacterial Activity: The antimicrobial activities of the synthesized polymers were tested using well cut diffusion method against gram positive Staphylococcus aureus and gram negative Escherichia coli. Each antimicrobial isolate was suspended in Mueller Hinton Agar (M-H) for performing antibiotic susceptibility tests and diluted to approximately $10^{5}$ Colony Forming Unit (CFU) per ml [17]. They were flood-inoculated onto the surface of (M-H) agar and then dried. Millimeter wells were cut from the agar using a sterile cork-borer and $30 \mu \mathrm{L}$ of the sample solution were poured into the wells and the plates were incubated for 18 hours at $37^{\circ} \mathrm{C}$. Antimicrobial activity was evaluated by measuring the diameter of the zone of inhibition in $\mathrm{mm}$ against the test microorganisms and the solvent DMSO was used as negative control and Ciprofloxacin was used as the reference for bacterial strains [18].

\section{RESULTS AND DISCUSSION}

A. Solubility: The Polymers poly (ortho-, meta-, and para) aminothiophenol were found to be completely soluble in DMSO, THF, DMF and benzene to give clear solutions and insoluble in water, methanol, n-hexane. They were partially soluble in ethanol and acetic acid.

B. FT-IR analysis: The FT-IR spectra of three polymers poly (o-aminothiophenol) (PoATP), poly (m-aminothio phenol) (PmATP) and poly (p-aminothiophenol) (PpATP) were given in figure 1. The participation of $\mathrm{N}-\mathrm{H}$ stretching vibration of the $-\mathrm{NH}$ group is confirmed by the single peak at $3379 \mathrm{~cm}^{-1}$. The peaks at 3379 and $3000 \mathrm{~cm}^{-1}$ were belonging to the asymmetric and symmetric $\mathrm{N}-\mathrm{H}$ stretching vibrations of $\mathrm{NH}_{2}$ group [19]. Two main peaks around 1620 and $1471 \mathrm{~cm}^{-1}$ in all the spectra correspond to the $\mathrm{C}=\mathrm{C}$ stretching vibrations of the quinoid and benzenoid rings [20]. The presence of these two rings clearly shows that the polymers are composed of the amine and imine units. The peak at $2601 \mathrm{~cm}^{-1}$ corresponds to S-H stretching. Furthermore the bands at 856 and $752 \mathrm{~cm}^{-1}$ were characteristic of C-H out-of plane bending vibrations of benzene nuclei. The strong peak at $1101 \mathrm{~cm}^{-1}$ is considered to be stemmed from delocalization of electrons in polymer backbone [21]. The PmATP, PpATP prepared have all the peaks of poly (oaminothiophenol) (PoATP) but the values are slightly shifted towards shorter wave number. 


\section{International Advanced Research Journal in Science, Engineering and Technology}

Vol. 5, Issue 8, August 2018

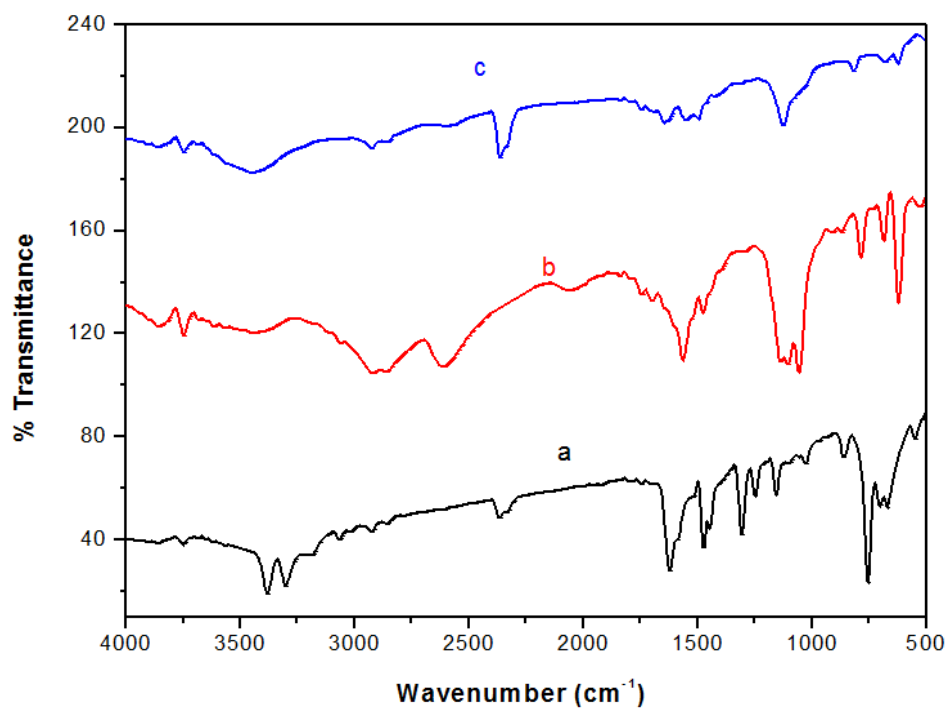

Fig.1 FT-IR spectra of (a) PoATP (b) PmATP (c) PpATP

C. UV-VIS Spectroscopy: The UV-VIS absorption spectra of the synthesised poly (ortho-, meta-, and para) aminothiophenol polymers dissolved in DMSO were given in figure 2. The UV-VIS spectra of PoATP, PmATP and PpATP consist of two major absorption peaks; the first peak at 250-270nm was assigned to the $\pi-\pi^{*}$ transition of the phenyl rings which is related to the extent of conjugation between the phenyl rings in the polymer chain. The intensity of the $\pi-\pi^{*}$ absorption maxima was comparable for all the three polymers [22]. The second absorption peak at 300$360 \mathrm{~nm}$ was assigned to $n-\pi *$ transition between the HOMO orbital of the benzenoid ring and the LUMO orbital of the quinoid ring and it was sensitive to the overall oxidation state of the polymer [23].

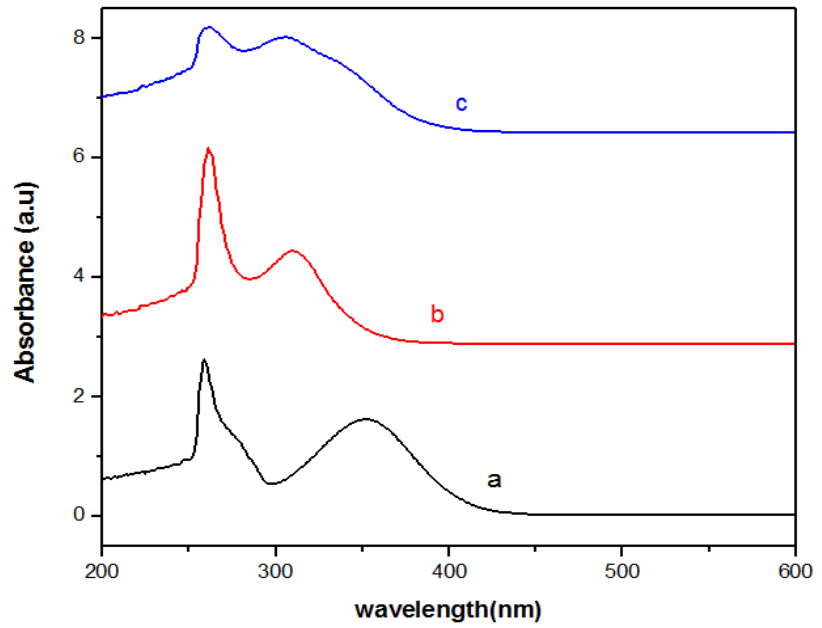

Fig.2 UV-Vis spectra of (a) PoATP, (b) PmATP (c) PpATP

D. XRD Analysis: Figure 3 shows the X-ray diffraction patterns of the synthesized polymers and the diffraction patterns were typical of crystalline/amorphous nature. The crystalline regions in the polymers were shown by the presence of relatively sharp peaks. The amorphous regions were visible by the broad low intensity halo; accordingly, the PoATP was more crystalline than PpATP and PmATP. The more amorphous nature of PpATP was inferred from their diffraction patterns. The position of the Bragg's peaks $(2 \theta)$ also gives information on the morphology of the polymers. All the polymers exhibited their strongest peaks at the $2 \theta$ values of $17.8^{\circ}, 19.7^{\circ}, 24.4^{\circ}, 25.6^{\circ}$ and the peak at $25.5^{\circ}$ was the characteristics of the van der Waals distances between stacks of phenylene rings [24]. These strongest peaks indicated the crystalline domains in the amorphous structure of the polymers. The degree of crystalline or ordered structural pattern in PoATP and PmATP was due to the more intrachain hydrogen bonding or electrostatic interaction which takes place through both amine and/or thiol group present in the polymer. The crystallinity and orientation of conducting polymers have been of much interest which influence the anticorrosion performance due to their metallic conductive state [25]. 


\section{International Advanced Research Journal in Science, Engineering and Technology}

Vol. 5, Issue 8, August 2018

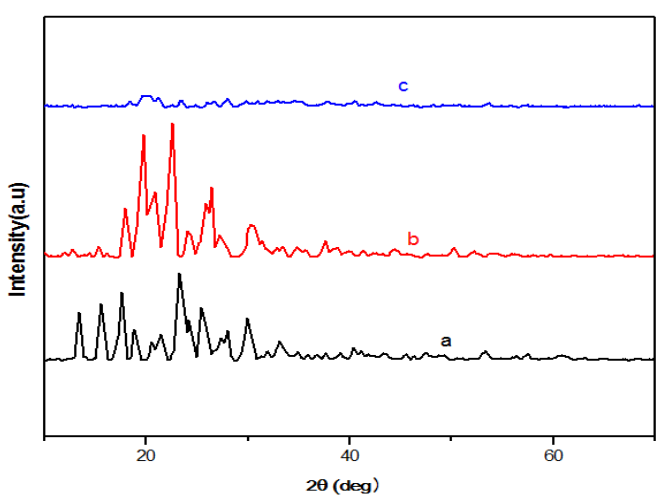

Fig.3 XRD pattern of (a) PoATP, (b) PmATP (c) PpATP

E. Thermal Analysis: The thermal stability of the poly (ortho-, meta-, and para) aminothiophenol polymers were evaluated using thermogravimetric analysis and their themograms were shown in figure 4.

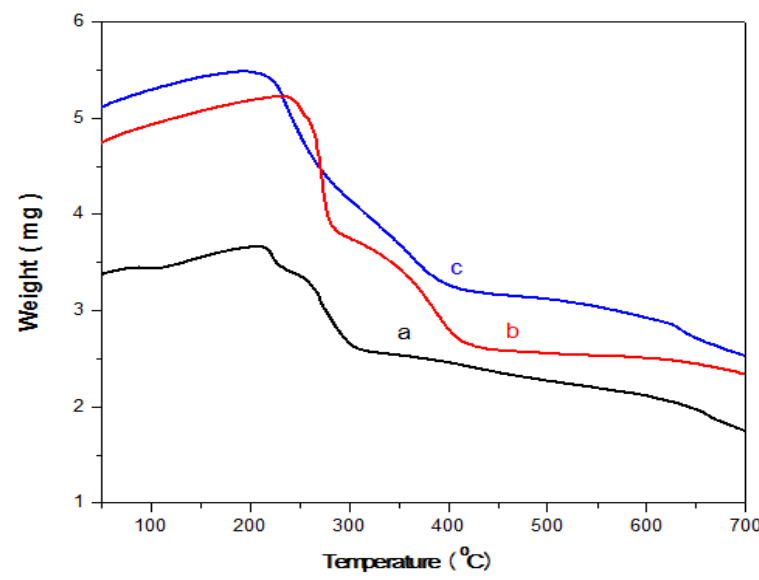

Fig. 4 TGA of (a) PoATP, (b) PmATP (c) PpATP

The thermal behaviour of the synthesized polymers were similar and exhibits three stage decomposition pattern. The first weight loss from $220^{\circ} \mathrm{C}$ to $400^{\circ} \mathrm{C}$ corresponds to the loss of free acids, and volatile molecules in polymer matrix. The second decomposition occured between and $400^{\circ} \mathrm{C}$ and $620^{\circ} \mathrm{C}$ due to loss of dopant, sublimation and removal of low molecular weight polymer/ oligomer from the polymer matrix [26]. While the third weight loss took place between $620^{\circ} \mathrm{C}$ and $732^{\circ} \mathrm{C}$ which was due to the complete degradation and decomposition of the polymer backbone. The decreasing degradation temperature order of synthesized polymers PpATP $\left(663^{\circ} \mathrm{C}\right)>\operatorname{PoATP}\left(637^{\circ} \mathrm{C}\right)>\operatorname{PmATP}$ $\left(430^{\circ} \mathrm{C}\right)$.

DTA curves of PoATP, PmATP and PpATP showed a wide peak with a plateau in temperature range of $427-750^{\circ} \mathrm{C}$ and this was related to the decomposition of organic moiety as shown in figure 5.The TGA and DTA study confirmed that the synthesized polymers possessed good thermal stability.

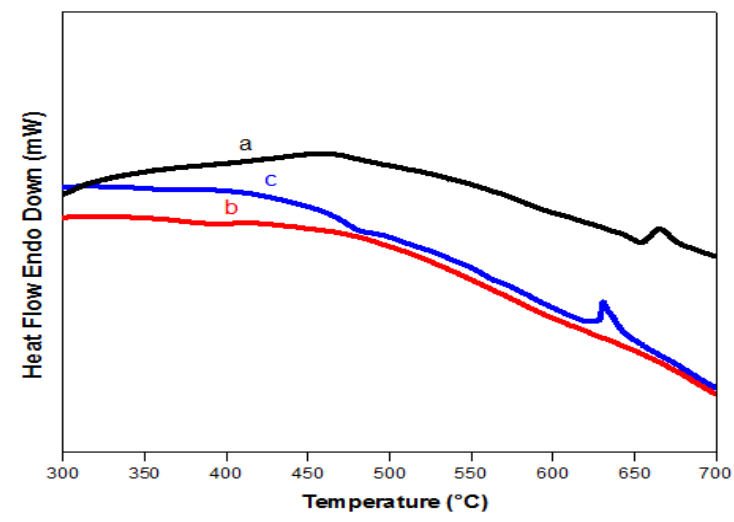

Fig. 5 DTA of (a) PoATP, (b) PmATP (c) PpATP 


\title{
International Advanced Research Journal in Science, Engineering and Technology
}

\author{
Vol. 5, Issue 8, August 2018
}

DSC can be used to obtain the thermal critical points like enthalpy specific heat or glass transition temperature(Tg). The DSC of poly (o-aminothiophenol), poly (m-aminothiophenol) and poly (p-aminothiophenol)) were given in figure 6. From the figure, it was found that the PoATP showed an endothermic peak at $92.73^{\circ} \mathrm{C}$ which is due to the glass transition temperature $(\mathrm{Tg})$ [27]. The peak at $261.56^{\circ} \mathrm{C}$ is due to the glass transition temperature ( $\mathrm{Tg}$ ) of PmATP and for PpATP it was $112.70^{\circ} \mathrm{C}$ [28].

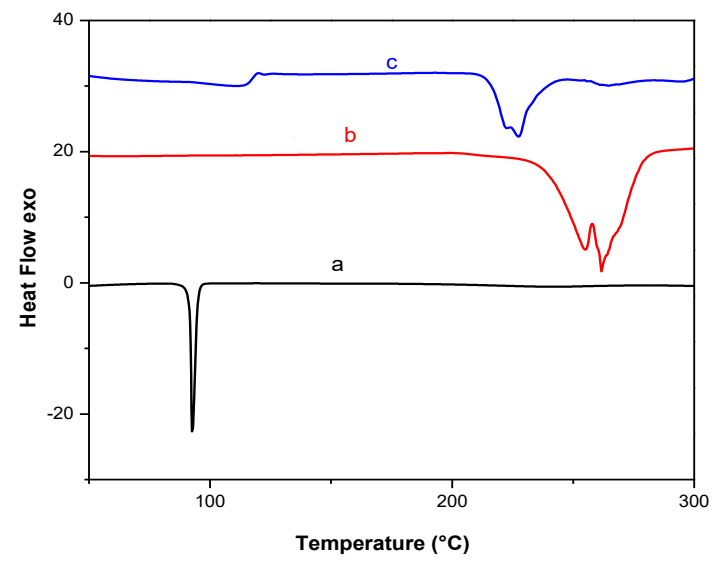

Fig. 6 DSC of (a) PoATP, (b) PmATP (c) PpATP

F. Scanning Electron Microscopy: The Scanning Electron Microscopy (SEM) analysis was carried out to study the morphology of the synthesized polymer. The morphology of chemically synthesized poly (o-aminothiophenol) (PoATP), poly (m-aminothiophenol) (PmATP) and poly (p-aminothiophenol) (PpATP) were shown in figures 7 -9 at different magnifications. The SEM image of PoATP (Figure 7) showed rod like arrangement, PmATP (Figure 8) exhibited agglomerated globular structure and PpATP figure (9) found to be in the smooth fiber form [29, 30]. The SEM analysis clearly showed the morphology of the polymers varied depending on the polymers.

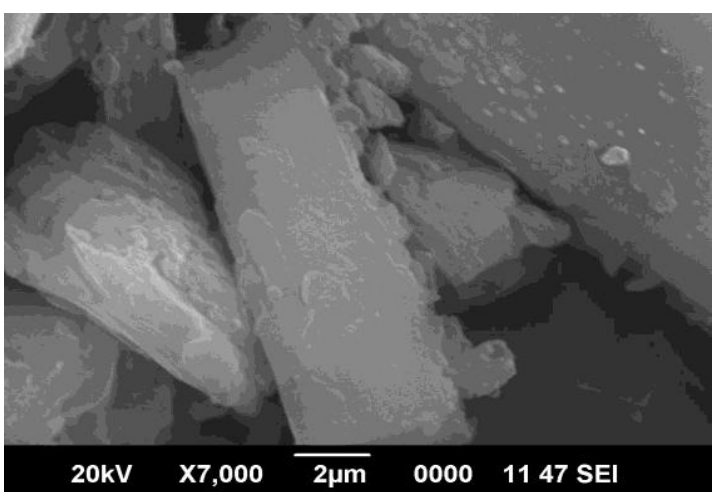

Fig.7 SEM image of PoATP

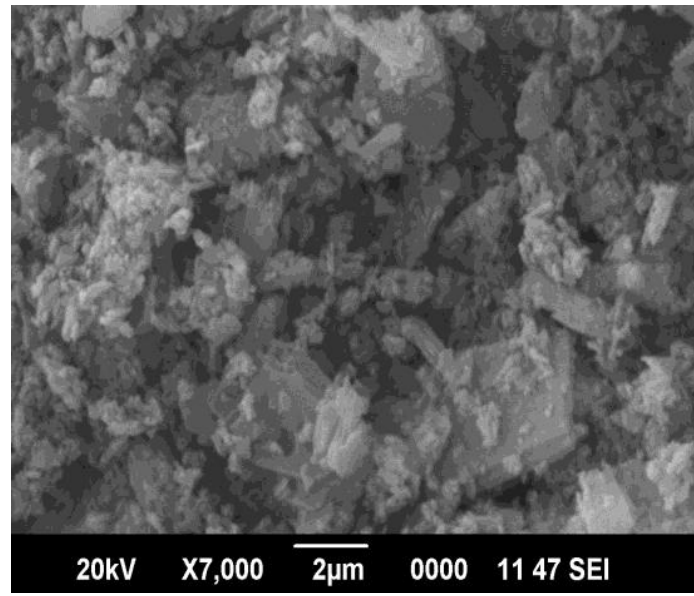

Fig. 8 SEM image of PmATP 


\section{International Advanced Research Journal in Science, Engineering and Technology}

Vol. 5, Issue 8, August 2018

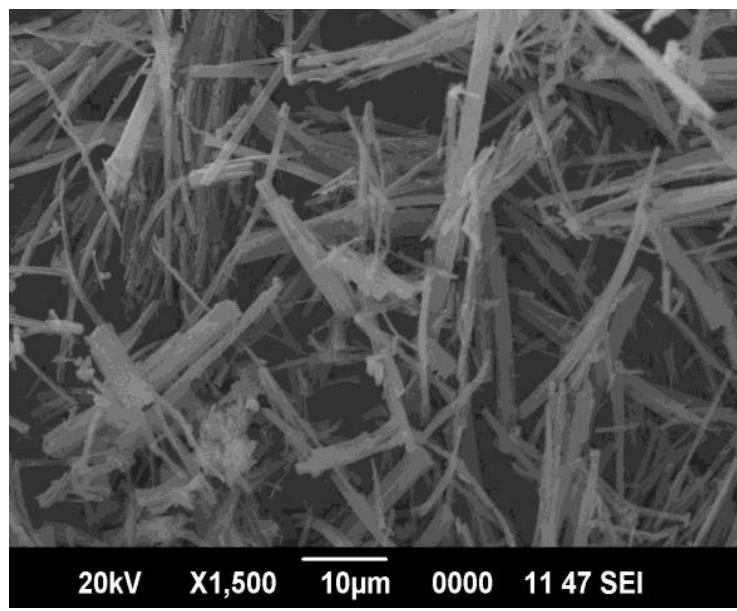

Fig.9 SEM image of PpATP

G. Electrical Conductivity: The electrical conductivity $\sigma$ was calculated [31] using the equation $\sigma=t /\left(R_{b} A\right)$ Where $\sigma$ is the ionic conductivity, $\mathrm{t}$ is the thickness of the sample, $\mathrm{A}$ is the most common area covered by the electrodes in contact with the sample, $R_{b}$ is the bulk resistance which is obtained from the intercept on the real axis at the high frequency end of the Nyquist plot at room temperature (303 K) were shown in (Figure 10) [32].

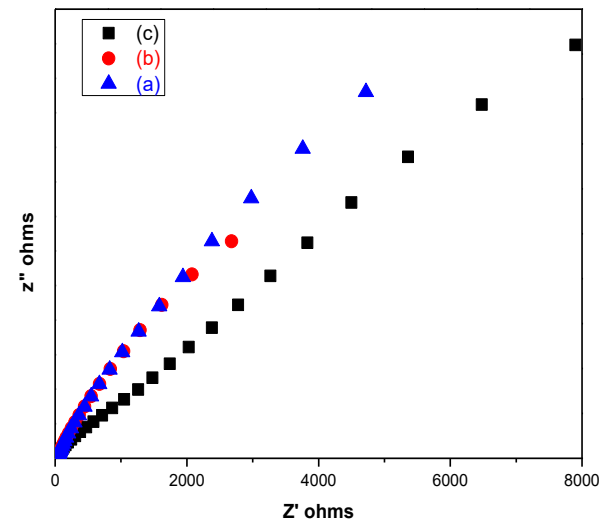

Fig.10 Nyquist plot of (a) PoATP (b) PmATP (c) PpATP

The ionic conductivities of the polymers PoATP, PmATP, PpATP were found to be $4.64 \times 10^{-4}, 4.34 \times 10^{-4}, 3.63 \times 10^{-}$ ${ }^{4} \mathrm{~S} / \mathrm{cm}$ respectively. The conductivity results showed that the polymers were semiconducting in nature and are almost similar and this may be due to the homogeneity in the crystalline/Amorphous nature of the polymer which was also evident from the XRD studies.

H. Antibacterial Activities: The antibacterial activities of the PoATP, PmATP and PpATP in different concentrations such as 50,75 and $100 \mu \mathrm{g} / \mathrm{ml}$ were investigated with gram positive bacteria like Staphylococcus aureus and gram negative bacteria like Escherichia Coli. Depending on the measured values with the zone of inhibition including the well in millimeter, the antibacterial activity can be classified into the following types: $>12 \mathrm{~mm}$ zone of inhibition - high sensitive, $9-12 \mathrm{~mm}$ zone of inhibition - moderate sensitive, $6-9 \mathrm{~mm}$ zone of inhibition - less sensitive and $<6 \mathrm{~mm}$ zone of inhibition - bacterial resistant [33-35].

The synthesized polymers found to inhibit the bacterial strains at different levels and their zone of inhibitions were given in table 1 . From the table values it was evident that PoATP were highly sensitive towards Staphylococcus aureus with the inhibition efficiencies of $17 \mathrm{~mm}, 18 \mathrm{~mm}, 20 \mathrm{~mm}$ and towards Escherichia coli the inhibition efficiency was found to be $16 \mathrm{~mm}, 21 \mathrm{~mm}, 22 \mathrm{~mm}$ which were comparable with the standard Ciprofloxacin whose inhibition efficiency was $22 \mathrm{~mm}$ towards Staphylococcus aureus and $18 \mathrm{~mm}$ for Escherichia coli and the sensitivity increased as the concentration of the polymers increased. The sample images of antibacterial activities of the poly (ortho-, meta-, and para) aminothiophenol against S.aureus were given in figure 11-13. 
International Advanced Research Journal in Science, Engineering and Technology

Vol. 5, Issue 8, August 2018

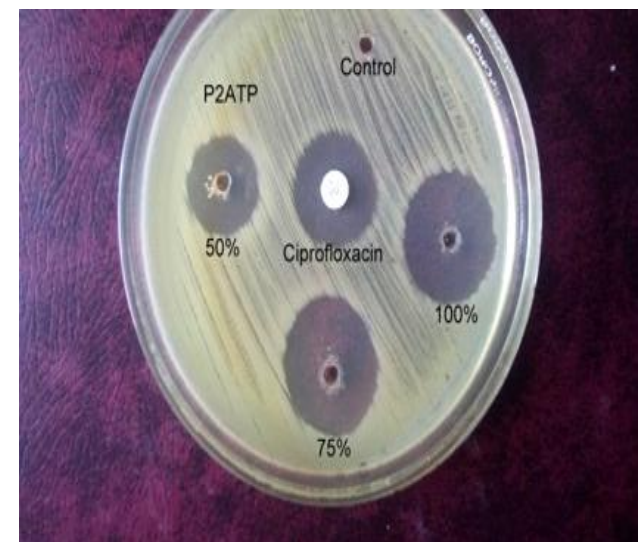

Fig.11 Sample image of antibacterial activity of PoATP against STAPHYLOCOCCUS AUREUS

Table I: Antibacterial Inhibition Efficiencies of Poly Aaminothiophenols

\begin{tabular}{|c|c|c|c|c|c|c|c|c|}
\hline \multicolumn{5}{|c|}{ Staphylococcus aureus } & \multicolumn{4}{|c|}{ Escherichia coli } \\
\hline Conc & $\begin{array}{l}\text { PoATP } \\
(\mathbf{m m})\end{array}$ & $\begin{array}{l}\text { PmATP } \\
(\mathbf{m m})\end{array}$ & $\begin{array}{c}\text { PpATP } \\
(\mathbf{m m})\end{array}$ & $\begin{array}{l}\text { Standard } \\
\text { Ciprofloxacin } \\
(\mathbf{m m})\end{array}$ & $\begin{array}{l}\text { PoATP } \\
(\mathbf{m m})\end{array}$ & $\begin{array}{l}\text { PmATP } \\
(\mathbf{m m})\end{array}$ & $\begin{array}{l}\text { PpATP } \\
(\mathbf{m m})\end{array}$ & $\begin{array}{l}\text { Standard } \\
\text { Ciprofloxacin } \\
(\mathbf{m m})\end{array}$ \\
\hline $50(\mu \mathrm{g} / \mathrm{ml})$ & 17 & 18 & 20 & 22 & 16 & 21 & 22 & 18 \\
\hline $75(\mu \mathrm{g} / \mathrm{ml})$ & 12 & 14 & 15 & 22 & 19 & 20 & 24 & 18 \\
\hline $100(\mu \mathrm{g} / \mathrm{ml})$ & 12 & 14 & 15 & 22 & 13 & 14 & 20 & 18 \\
\hline
\end{tabular}

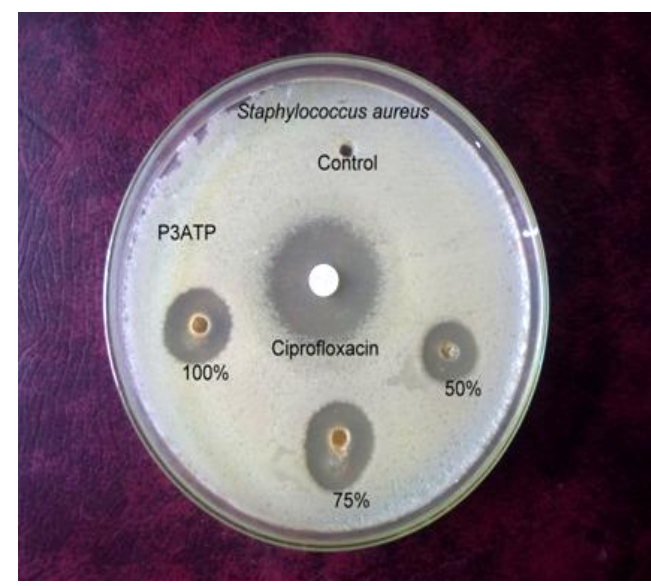

Fig.12 Sample image of antibacterial activity of PmATP against STAPHYLOCOCCUS AUREUS

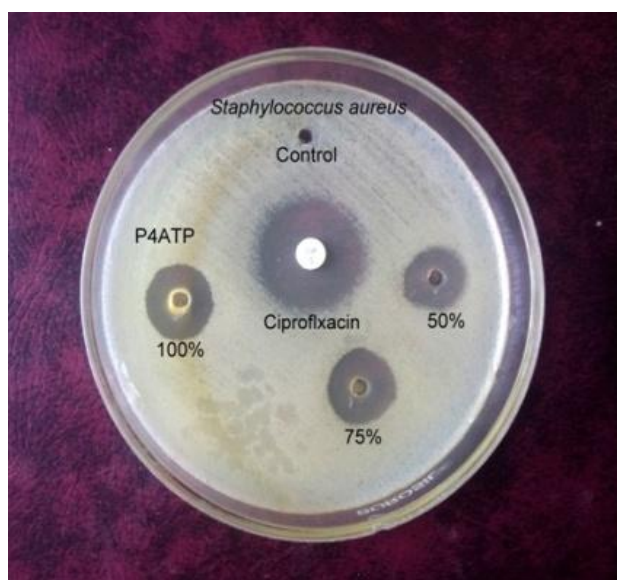

Fig.13 Sample image of antibacterial activity of PpATP against STAPHYLOCOCCUS AUREUS 


\title{
International Advanced Research Journal in Science, Engineering and Technology
}

\author{
Vol. 5, Issue 8, August 2018
}

The antibacterial activities of PmATP was found to be $12 \mathrm{~mm}, 14 \mathrm{~mm}$ and $15 \mathrm{~mm}$ towards Staphylococcus aureus and $19 \mathrm{~mm}, 20 \mathrm{~mm}, 24 \mathrm{~mm}$ against gram negative bacteria Escherichia coli at different concentrations of PmATP like 50, 75, $100 \mu \mathrm{g} / \mathrm{mL}$ The sample images of antibacterial activities of the synthesized polymers like poly (ortho-, meta-, and para) aminothiophenol against E.Coli were given in figure 14-16. The inhibition efficiency of PpATP towards Staphylococcus aureus and Escherichia coli were found to be $12 \mathrm{~mm}, 14 \mathrm{~mm}, 15 \mathrm{~mm}$ and $13,14,20 \mathrm{~mm}$ at different concentrations of the polymer and it was comparable with the standard Ciprofloxacin whose bacterial inhibition efficiencies were found to be $22 \mathrm{~mm}$ and $18 \mathrm{~mm}$ towards gram positive and gram negative bacterial strains.

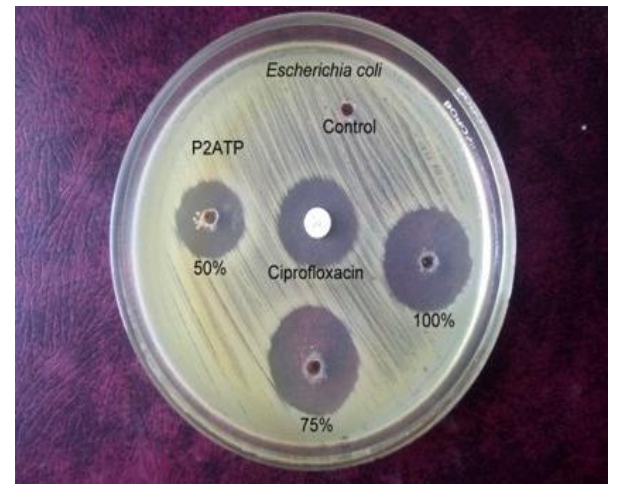

Fig.14 Sample image of antibacterial activity of PoATP against Escherichia coli

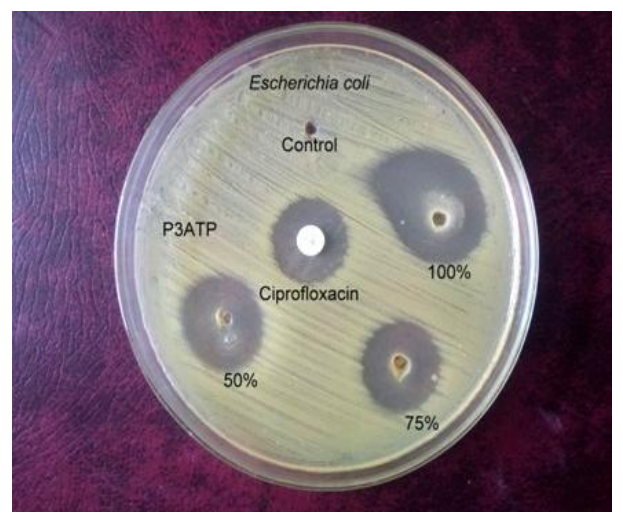

Fig.15 Sample image of antibacterial activity of PmATP against Escherichia coli

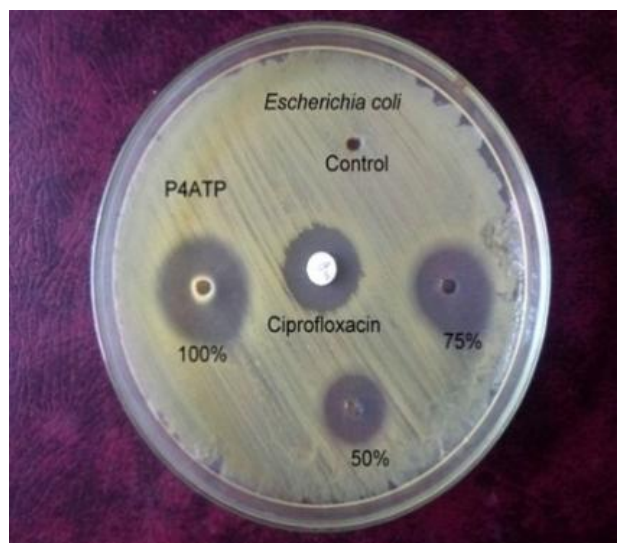

Fig.16 Sample image of antibacterial activity of PpATP against Escherichia coli

At the highest concentration of $100 \mu \mathrm{g} / \mathrm{ml}$, the bacterial inhibition efficiency was found to be the highest with the inhibition efficiency of $20 \mathrm{~mm}$ against Staphylococcus aureus and it is found to decrease in the order PoATP $>$ PmATP $>$ PpATP and for Escherichia coli, the highest inhibition efficiency was found to be $24 \mathrm{~mm}$ for PmATP and the activity found to decrease in the order PmATP $>$ PoATP $>$ PpATP. From the table it was evident that the sensitivity increases with increase in concentration. 


\title{
International Advanced Research Journal in Science, Engineering and Technology
}

\author{
Vol. 5, Issue 8, August 2018
}

\section{CONCLUSION}

The isomers of poly aminothiophenols like poly (o-aminothiophenol), poly ( $\mathrm{m}$ aminothiophenol) and poly (paminothiophenol) were successfully synthesized by chemical oxidative polymerization method and characterized by different spectroscopic techniques. FT-IR suggested the formation of quinoid and benzenoid rings by their stretching vibrations and UV-VIS spectra confirmed the formation of polymer with the $\pi-\pi^{*}$ and $n-\pi^{*}$ transitions. The XRD studies revealed isomers of poly (aminothiophenol) were partially crystalline and amorphous nature. The thermal stabilities of the poly (ortho-, meta-, and para) aminothiophenol were confirmed from TGA /DTA and DSC studies and found that the polymers were more stable up to $700^{\circ} \mathrm{C}$. The morphologies of the polymers studied by SEM analysis showed the rod, globular and fiber like structures for PoATP, PmATP and PpATP. The electrical conductivities of the polymers showed that they were semiconducting in nature and the conductivity was higher in the order of $10^{-4}$. The present investigations revealed that the isomers of poly (aminothiophenol) have excellent antibacterial activities, further their compatibility in biological studies will give fabulous scope of these synthetic polymers in biomedical applications. Further research could pave way for the development of novel drugs to control diseases and infections. The synthesized polymers can be used in different applications in furture. In addition, the $\pi$-electrons conjugation, quaternary nitrogen atom, and large size of the polymer give hope to facilitate their strong adsorption on Mild steel (MS) which may lead to an efficient protection agent to the metals in highly corrosive medium and this will favour the protection of the metals from the corrosive environment.

\section{REFERENCES}

[1]. Kondawar, S. B.; Thakare, S. R.; Bompilwar, S.; Khati, V. Int. J. Mod. Phys. B. 2009,23, 3297.

[2]. Lei, J.; Menon, V.P.; Martin, C.R. Polym. Adv. Tech., 1993, 41, 24.

[3]. Yang, C.H.; Wang, T.L.; Shieh, Y.T.; Electrochemistry Communications, 2009, 11,335

[4]. Horie, M.; Yamaguchi, I.; Yamamoto, T. Macromolecules., 2006, 39, 7493.

[5]. Jude, O.I. and Kirill L.,J. Euro. Polym.,2002, 38, 1547-1550.

[6]. Shukla,S.K.; Bharadvaja, A.; Tiwari, A.; Parashar, G.K.; Dubey, G.C. Adv. Mat. Lett. 2010, 1(2), 129.

[7]. Gupta, M. C.; Borkar, A. D.; Umare, S. S.; Polym. Plast. Technol. Eng. 2001, 40, 225.

[8]. Kumari, K.; Ali, V.; Rani, G.; Kumar, S., Lakshmi, G., Zulfequar, M. Materials Science and Application ,2011, 2, 1047-1057

[9]. Friend, R. H.; Gymer, R. W.; Holmes, A. B.; Nature, 1997 , 397, 121-128.

[10]. Saraswathi, R., Gerard, M. Malhotra, B.; Journal of Applied Polymer Science, 1999, 74,145.

[11]. Friend, R. H.; Gymer, R. W.; Holmes, A. B.; Nature, 1997 , 397, 121-128.

[12]. Saber-Tehrani, M.; Pourhabib, A.; Husain, S.W.; Arvand, M. Food Anal. Methods, 2013, 6, 1300-1307.

[13]. Zhang, L.;Jia, J.;Zou, X.;Dong, S. Electroanalysis., 2004, 16, 1413-1418.

[14]. Gopalan, A.I.; Lee, K.-P.; Manesh, K.M.; Santhosh, P.; Kim, J.H.; Kang, J.S. Talanta., 71, (2007), 1774-1781

[15]. Marija Gizdavic; Jadranka Travas-Sejdic; Graham A Bowmaker; Ralph P Cooney; Corrina Thompson; Paul AKilmartin. Current Applied Physics., 2004, 4,347-350

[16]. Irudaya Antonat Sophia, G. Gopu, C. Vedhi., Open Journal of Synthesis Theory and Applications., ( 2012), 1, 1-8.

[17]. Mahito Atobe;Al-Nakib Chowdhury;Toshio Fuchigami; Tsutomu Nonaka. Ultrasonics Sono-chemistry, 2003,10,77-80

[18]. L Estevinho; AP Pereira; L Moreira; G Luis. Food. Chem. Toxicol., 2008, 46, 3774

[19]. Qingli Hao. Baoming Sun. Xujie Yang. Lude Lu. Xin Wang. Materials Letters 2009: 63: 334-336.

[20]. M.R. Nabid, R. Sedghi, P.R. Jamaat, N. Safari, A.A. Entezami, Appl. Catal. A., 2007 ,328 , $52-57$.

[21]. M.R. Nabid, R. Sedghi, A. Bagheri, M. Behbahani, M. Taghizadeh, H. Abdi Oskooie, M.M.Heravi, J. Hazard. Mater., 2012 , 203 93.

[22]. S. K. Shukla, M. A. Quraishi and R. Prakash, Corrosion Science, 2008, Vol. 50, No. 10, pp. 2867-2872.

[23]. A. Elmansouri, A. Outzourhit, A. Lachkar et al., SyntheticMetals, 2009, vol. 159, no. 3- 4,pp. 292-297,

[24]. C. Saravanan, S. Palaniappan, and F. Chandezon, Materials Letters, vol. 62, no. 6-7, pp. 882-885, 2008.

[25]. H. Xia and Q. Wang, Chemistry of Materials,, 2002 vol. 14, no. 5, pp.2158-2165.

[26]. C. Sivakumar, T.C.Wen, A. Gopalan, and H.Teng, Synthetic Metals, 2003, 132, 219- 226.

[27]. Alan Riga. Ricardo Collins. Gregory Mlachak., Thermochimica Acta. 1998: 324: 135- 149.

[28]. Ossama M.Abo-Elenien. Ashraf M. Elsaeed. Maher A. El-Sockary. Int. Journal of Engineering Researchand Applications.2014:4:148-155.

[29]. S. Murlidharan, K. L. N. Phani, S. Pitchumani, S. Ravi-chandran and S. V. K. Iyer, Journal of the Electrochemical Society, 1995, Vol. 142, No. 5, pp. $1478-1483$

[30]. S. R. Kargirwar,S. R. Thakare, M. D. Choudhary, D. K. Burghate., Int.J.Nano Dim., 2012, 2(4), $223-226$.

[31]. Porselvi Linganathan, Jhancy Mary Samuel, American Journal of polymer Science., 2014, 4, 107-116.

[32]. Ganesan, S.; Muthuraaman, B.; Vinod Mathew.; Madhavan, J.; Maruthamuthu, P.; Austin Suthanthiraraj, S. Sol. Energy Mater. Sol. Cells, 2008, 2,1718.

[33]. D Sai Koteswar Sarma;A Venkata Suresh Babu;K.Rama Krishna;PP Nagoor Basha. J.Chem. Phar. Res., 2011, 3(4), 875-881.

[34]. Firdaus Jahan;Rubina Lawrence;Vinod Kumar;Mohd Junaid. J.Chem .Pharm Res.,2011, 3 (4), 777-789

[35]. R Kavitha ;P Kamalakannan; T Deepa, R Elamathi;S Sridhar;J Suresh Kumar. J.Chem .Pharm. Res., 2011, 3(6),115-121 